\title{
Efficacy and safety of once-monthly injection of paliperidone palmitate in hospitalized Asian patients with acute exacerbated schizophrenia: an open-label, prospective, noncomparative study
}

\author{
This article was published in the following Dove Press journal: \\ Neuropsychiatric Disease and Treatment \\ 21 December 2015 \\ Number of times this article has been viewed
}

\section{HuaFang Li' \\ Ibrahim Turkoz ${ }^{2}$ \\ Fan Zhang ${ }^{3}$}

'Shanghai Mental Health Center, Shanghai Jiao Tong University School of Medicine, Shanghai, People's Republic of China; ${ }^{2}$ Janssen Research \& Development, LLC, Titusville, NJ, USA; ${ }^{3}$ Xi'an Janssen Pharmaceutical Ltd., Beijing, People's Republic of China
Correspondence: Ibrahim Turkoz Director Biostatistics, Janssen

Research \& Development LLC, I 25

Trenton-Harbourton Road, Titusville,

NJ 08560, USA

Tel +l 6097307719

Fax + I 6097303232

Email ITurkoz@its.jnj.com
Introduction: This single-group, open-label, prospective, noncomparative, multicenter, Phase IV study explored the efficacy and tolerability of paliperidone palmitate (PP) in hospitalized patients with acute exacerbation of schizophrenia.

Methods: Asian patients of either sex, between 18 and 65 years of age, diagnosed with schizophrenia (Diagnostic and Statistical Manual of Mental Disorders, Fourth Edition) with acute exacerbations within the previous 4 weeks, were enrolled. Intramuscular PP was initiated at doses of 150 milligram equivalent (mg eq) (day 1) and $100 \mathrm{mg}$ eq (day 8), followed by a monthly maintenance dose between $75 \mathrm{mg}$ eq and $150 \mathrm{mg}$ eq (days 36 and 64). Primary efficacy endpoint was the change from baseline in the Positive and Negative Syndrome Scale (PANSS) total score (last-observation-carried-forward) at week 13.

Results: Of the 212 enrolled patients, 152 (71.7\%) completed the 13-week treatment; withdrawal of consent (24 [11.3\%] patients) was the most common reason for study discontinuation. Mean (standard deviation) PANSS total score from baseline (90.0 [17.41]) improved significantly at day 4 (-6.1 [9.27]; 95\% confidence interval: $-7.38,-4.85 ; P<0.001)$ and week 13 endpoint ( -23.9 [23.24]; 95\% confidence interval: $-27.10,-20.78 ; P<0.001)$. Similarly, the secondary endpoints (Clinical Global Impression-Severity, Physical and Social Performance, each PANSS subscale, and Marder factor scores) improved significantly from baseline to week 13 endpoint ( $P<0.001$ for all). At week 13, 112/210 (53.3\%) patients had a 40\% improvement in the PANSS total score (responder rate), and 133/212 (62.7\%) patients were ready for hospital discharge. Overall, $139(65.6 \%)$ patients experienced at least one treatment-emergent adverse event (TEAE). Most common ( $>5 \%$ ) TEAEs were hyperprolactinemia, constipation, nasopharyngitis, insomnia, increased weight, and tremor. Worsening of schizophrenia (3.3\%) and sinus bradycardia $(2.0 \%)$ were serious TEAEs; no deaths were reported.

Conclusion: PP was generally tolerable and efficacious in a hospital setting for the treatment of acute exacerbated schizophrenia with significant improvements in psychotic symptoms, social functioning, and severity of illness.

Keywords: paliperidone palmitate, exacerbation, Asian, hospital, acute schizophrenia

\section{Introduction}

Schizophrenia is one of the most common diagnosis among the psychiatric inpatients. ${ }^{1-3}$ The majority ( $85 \%-90 \%)$ of the patients diagnosed with schizophrenia demonstrate exacerbations. ${ }^{4}$ Acute treatment of schizophrenia facilitates the management of symptom exacerbation, enhances psychosocial functioning, and improves the quality of life. ${ }^{5}$ Furthermore, continuous, integrated, long-term pharmacotherapy is required 
to provide relief from psychotic symptoms and to reduce the relapse rates. ${ }^{5,6}$

Long-term treatment strategies may result in a risk of treatment nonadherence, generally because of associated adverse outcomes and the higher cost of care. ${ }^{7-9}$ Treatment nonadherence in the individuals with schizophrenia may lead to acute worsening of psychotic and behavioral symptoms, requiring multiple hospitalizations. ${ }^{5,10,11}$ Long acting injectable (LAI) formulations of antipsychotics exert advantages over oral antipsychotic treatment by improving adherence, reducing symptoms, risk of relapse, and rehospitalization. ${ }^{12-15}$

Among the various available LAI antipsychotics, paliperidone palmitate (PP) (Invega ${ }^{\circledR}$ Sustenna ${ }^{\circledR}$ ), a once-monthly intramuscular injection, ${ }^{16}$ is approved in the USA as well as in most of the Asia Pacific countries for the acute and maintenance treatment of adults with schizophrenia. ${ }^{17,18}$

The use of LAIs has been limited to the maintenance treatment or prevention of relapse, and because of their longer time to achieve steady state, effectiveness in the acute treatment phase may require additional oral supplementation. ${ }^{19-21}$ The LAI PP has a unique pharmacokinetic profile, which allows both, a rapid achievement of therapeutic plasma levels of paliperidone and a gradual and continuous release of the drug over the dose administration interval. ${ }^{18,21,22}$ Such a profile permits the initiation of PP in acutely symptomatic patients without oral supplementation, with demonstrated efficacy and tolerability across the dose range of $50-150 \mathrm{mg}$ eq. ${ }^{7,17,18,23}$

To date, very few studies have been designed to investigate the use of $\mathrm{PP}^{24}$ or other LAI antipsychotics ${ }^{20,25-27}$ as acute therapy for patients who are hospitalized due to a recent exacerbation of schizophrenia. Therefore, the current study (PREVAIL; A Study to Evaluate the Effectiveness and Safety of Paliperidone Palmitate in Subjects With Acute Schizophrenia, NCT01527305) was conducted to investigate the efficacy and safety of PP for the treatment of Asian hospitalized patients with schizophrenia who have experienced an acute exacerbation within the past 4 weeks of hospitalization. The use of concomitant medications, including antipsychotics, in treating patients with acute schizophrenia was also investigated.

\section{Methods}

\section{Study population}

Asian patients of either sex, between 18 and 65 years (inclusive) of age, diagnosed with schizophrenia according to the Diagnostic and Statistical Manual of Mental Disorders, Fourth Edition (DSM-IV), with a Positive and Negative
Syndrome Scale (PANSS) total score of $\geq 60$, or Clinical Global Impression-Severity (CGI-S) score of $\geq 4$ (moderately ill), and hospitalized with an acute exacerbation of schizophrenia within the previous 4 weeks of screening, were included. The key exclusion criteria were the following: primary active DSM-IV Axis I diagnosis other than schizophrenia, DSM-IV diagnosis of substance dependence (except for nicotine and caffeine) within 6 months of screening, patients who received clozapine, or LAI antipsychotics including PP within 1 month of the screening, and those who were at a significant risk of suicide.

The study protocol was reviewed and approved by the local study site's Institutional Review Board, Medical Ethics Committee, or Medical Research and Ethics Committee in the participating countries. This study was conducted in accordance with the ethical principles that have their origin in the Declaration of Helsinki, and in accordance with the International Conference on Harmonization's Good Clinical Practice guidelines, applicable regulatory requirements, and in compliance with the respective protocols. All patients provided written informed consent prior to study participation.

\section{Study drugs}

As the doses of PP can be expressed both in terms of milligram equivalent (mg eq) of the pharmacologically active fraction, ie, paliperidone, and in milligrams of PP, the doses expressed as 75,100 , and $150 \mathrm{mg}$ eq equate to 117,156 , and $234 \mathrm{mg}$ of paliperidone palmitate, respectively. The LAI PP $\left(\right.$ Invega ${ }^{\circledR}$ Sustenna $^{\circledR}$ ) was supplied as prefilled syringes of unrefrigerated suspension for intramuscular injection at doses of 75,100 , or $150 \mathrm{mg}$ eq. Deltoid injections were administered using a 1.5-inch needle for patients weighing $\geq 90 \mathrm{~kg}$, and a 1 -inch needle for patients weighing $<90 \mathrm{~kg}$, while the gluteal injections were administered using a 1.5 -inch needle.

\section{Study design}

This single-group, open-label, prospective, noncomparative, Phase IV study (NCT01527305) was conducted at 27 centers in four Asian countries (six sites in People's Republic of China, ten in Korea, three in Malaysia, and eight in Taiwan) from June 13, 2012 to December 31, 2013. The study consisted of a screening period up to 7 days followed by a 13-week open-label treatment period, and a study completion visit on day 92 (week 13, 1 month after the last dose of the PP injection). All patients were switched to PP from their existing oral antipsychotic medication on day 1. Patients without documented tolerability to oral risperidone, extendedrelease (ER) paliperidone, risperidone-LAI, or PP were 
administered with paliperidone ER $3 \mathrm{mg} /$ day or $1 \mathrm{mg}$ /day oral risperidone for at least 2 days for the oral tolerability testing during the screening period. In the open-label treatment period, patients received PP at an initiation dose of $150 \mathrm{mg}$ eq on day 1 and $100 \mathrm{mg}$ eq on day 8 (both deltoid), followed by once-monthly maintenance doses of 75,100 , or $150 \mathrm{mg}$ eq on days 36 and 64 (deltoid or gluteal).

\section{Concomitant medications}

Medications for general medical conditions were allowed. Additionally, mood stabilizing and antidepressant medication (except nonselective or irreversible monoamine oxidase inhibitors), $\beta$-blockers, sedative-hypnotics, antiparkinsonian medication with anticholinergic properties (in case of emergence or worsening of extrapyramidal symptoms [EPS]), benzodiazepines (as rescue medication at the maximum permitted daily doses for $\leq 10$ consecutive days during a 1-month interval), and oral lorazepam (up to $6 \mathrm{mg}$ /day) or equivalent benzodiazepine, for the control of agitation, irritability, restlessness, and hostility, were allowed.

\section{Study assessments}

\section{Efficacy assessments}

The primary efficacy variable was the change in the PANSS total score from baseline to the end of the open-label period. The secondary efficacy variables included the changes from baseline to the endpoint in the PANSS responder rate (percentage of patients with $\geq 40 \%$ reduction in PANSS total score), PANSS Marder factors (negative symptom, positive symptom, disorganized thought, uncontrolled hostility/ excitement, and anxiety/depression), ${ }^{28}$ PANSS subscales (positive, negative, or general psychopathology), CGI-S scale, ${ }^{29}$ personal and social performance (PSP) scale scores, ${ }^{30}$ the time to readiness for hospitalization discharge using the ready-to-discharge questionnaire (RDQ), ${ }^{31,32}$ and the rate of drug discontinuation.

The CGI-S was administered to assess the severity of the disease. In this study, the CGI-S wording was modified to match the protocol description from 1 to 7 where $1=$ normal, not at all ill, $2=$ borderline mentally ill, $3=$ mildly ill, $4=$ moderately ill, 5= markedly ill, $6=$ severely ill, and $7=$ extremely severely ill.

The CGI-S and RDQ assessments were performed at screening, days 1, 8, 36, 64, and 92, and the PSP was administered on days 1 and 92. The PANSS total score, Marder factor score, subscales score, and responder rate were analyzed at screening, and days 1, 4, 8, 36, and 92. All the efficacy assessments were administered and evaluated by independent, trained raters, at each site, to reduce inconsistencies in the data collection, and evaluation of the clinical endpoints. Lower scores in the PANSS total, PANSS subscale, Marder factor, and CGI-S scores indicated improvement, whereas, higher scores indicated improvement in the PSP scores.

\section{Safety assessments}

Safety assessments included the incidence, and the type of treatment-emergent adverse events (TEAEs), concomitant use of psychotropic medications, clinical laboratory tests, physical examinations (including body weight, height, and body mass index [BMI]), vital sign measurements, and 12-lead electrocardiograms.

\section{Sample size determination}

A sample size of 185 patients was considered sufficient to provide $90 \%$ power to detect a clinically meaningful difference of at least 6 points in the mean change from baseline to the endpoint in the PANSS total score with an estimated standard deviation (SD) of 25 units, and a significance level of 0.05 , using a two-sided one-sample $t$-test. Assuming a $20 \%$ dropout, 232 patients were to be enrolled to ensure that at least 185 participants completed the study.

\section{Analysis sets}

All efficacy and safety analyses were performed on the intent-to-treat (ITT) analysis set, which included patients who received at least one dose of the study medication during the treatment period. Additional supportive analyses were performed on the per-protocol set, which included patients without major protocol deviations. Major protocol deviation was defined as patients not satisfying the inclusion criteria; who developed withdrawal criteria during the study but did not withdraw from the study; who received the wrong treatment or incorrect dose; and those patients who received an excluded concomitant treatment. A post hoc confirmatory analysis of efficacy excluding all patients who had received a concomitant antipsychotic medication after day 1 was also performed.

\section{Statistical analyses}

Demographic and baseline characteristics were summarized descriptively. Efficacy variables were also summarized descriptively and examined using paired $t$-test or Wilcoxon signed-rank test as appropriate. Time-to-readiness for discharge and time to the first actual discharge were analyzed using Kaplan-Meier methodology. In addition, time to discharge was examined using Cox proportional hazards 
regression models, including the baseline characteristics as covariates. The rate of drug discontinuation was summarized by the number and percentage of patients who were discharged from the hospital. No adjustment was made for multiplicity. The last observation carried forward (LOCF) approach was used to impute the missing data in the analysis set. The number of patients with at least one TEAE for each preferred term was summarized regardless of the severity and relationship to the study medication. Results of clinical laboratory parameters and vital signs were summarized using descriptive statistics.

\section{Results}

\section{Patient disposition and demographics}

Of the 212 patients in the ITT analysis set, $152(71.7 \%)$ completed the 13 -week treatment period, with the most common reason for study discontinuation being withdrawal of consent (24 [11.3\%] patients) (Figure 1). The study population was $100 \%$ Asian and balanced by sex. The mean (SD) age of the population was 37.1 (11.8) years, and the BMI was 23.6 (4.24) kg/m² (Table 1). Demographic and baseline characteristics were similar between the ITT and the per-protocol populations.

In this study, paranoid schizophrenia was the most frequent $(70.3 \%)$ schizophrenia type. Majority of the patients $(89.2 \%)$ had a baseline PANSS score of $\geq 70$. As per CGI-S score, $70 \%$ of the patients were marked or severe or extremely severely ill, and as per PSP score, $23.6 \%$ patients had poor level of functioning that required intensive supervision.

Prior psychotropic medications were used by 168 (79.3\%) patients. The most commonly ( $\geq 20 \%$ ) used antipsychotics were risperidone (77 [36.3\%] patients), lorazepam (57 [26.9\%] patients), and paliperidone (49 [23.1\%] patients).

The mean (SD) dose of PP was 122.0 (13.63) $\mathrm{mg}$ eq throughout the study, while that from the third dose to the last dose was 114.5 (25.52) $\mathrm{mg}$ eq. Overall, 77 (36.3\%) patients received the last dose as $150 \mathrm{mg}$ eq, $120(56.6 \%)$ patients as $100 \mathrm{mg}$ eq, and $15(7.1 \%)$ patients as $75 \mathrm{mg}$ eq. The duration of PP exposure was $\geq 92$ days for $63(29.7 \%)$ patients, 64-91 days for 104 (49.1\%) patients, 36-63 days for $17(8.0 \%)$ patients, 8-35 days for $19(9.0 \%)$ patients, and $\leq 7$ days for nine $(4.2 \%)$ patients. Of the $90(42.5 \%)$

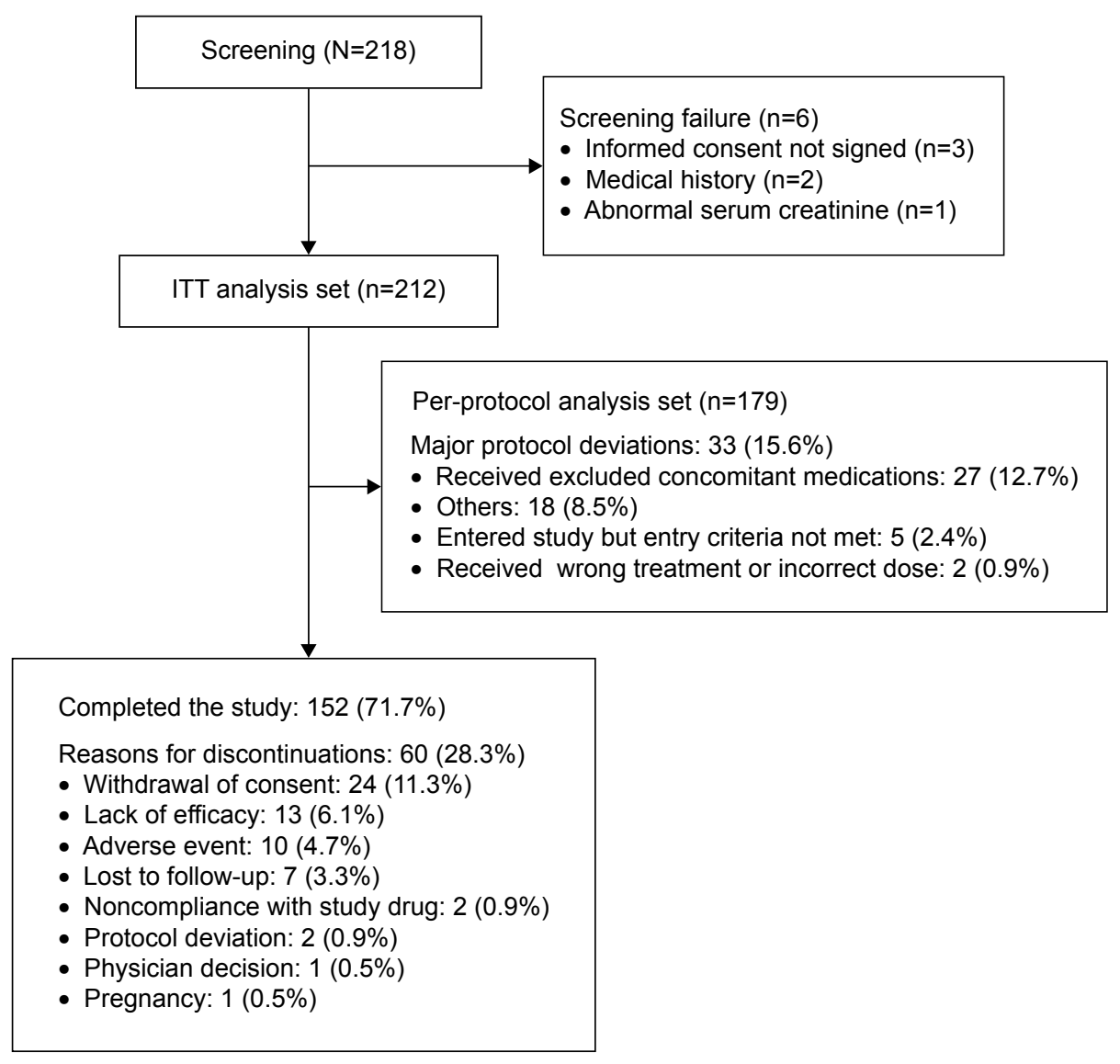

Figure I Patients' disposition. Abbreviation: ITT, intent-to-treat. 
Table I Demographic and baseline characteristics (ITT analysis set)

\begin{tabular}{|c|c|}
\hline Parameter & All patients $(\mathrm{N}=2 \mid 2)$ \\
\hline Age in years, mean $(S D)$ & $37.1($ (II.82) \\
\hline Age range in years & $18-64$ \\
\hline I8-25 years, $\mathrm{n}(\%)$ & $43(20.3)$ \\
\hline $26-50$ years, $n(\%)$ & $133(62.7)$ \\
\hline$>50$ years, $n(\%)$ & $36(17.0)$ \\
\hline \multicolumn{2}{|l|}{ Sex, n (\%) } \\
\hline Men & $108(50.9)$ \\
\hline Women & $104(49.1)$ \\
\hline $\mathrm{BMI}, \mathrm{kg} / \mathrm{m}^{2}$, mean (SD) & $23.6(4.24)^{\mathrm{a}}$ \\
\hline \multicolumn{2}{|l|}{ Category, n (\%) } \\
\hline Normal weight $<25$ & $147(69.7)$ \\
\hline Overweight 25 to $<30$ & $47(22.3)$ \\
\hline Obese $\geq 30$ & $17(8.1)$ \\
\hline $\begin{array}{l}\text { Age at first schizophrenia diagnosis, } \\
\text { mean (SD) years }\end{array}$ & $29.7(11.21)$ \\
\hline \multicolumn{2}{|l|}{ Schizophrenia type, n (\%) } \\
\hline Paranoid & $149(70.3)$ \\
\hline Disorganized & $10(4.7)$ \\
\hline Undifferentiated & $50(23.6)$ \\
\hline Residual & $3(1.4)$ \\
\hline Baseline PANSS total score, mean (SD) & $90.0(|7.4|)$ \\
\hline Baseline CGI-S score, mean (SD) & $4.9(0.79)$ \\
\hline Mild illness, n (\%) & $6(2.8)$ \\
\hline Moderate illness, n (\%) & $59(27.8)$ \\
\hline $\begin{array}{l}\text { Marked, severe/extremely severe } \\
\text { illness, n (\%) }\end{array}$ & $147(69.3)$ \\
\hline
\end{tabular}

Note: ${ }^{\mathrm{a}} \mathrm{n}=2 \mathrm{I}$ I.

Abbreviations: BMI, body mass index; CGI-S, Clinical Global Impression-Severity; ITT, intent-to-treat; PANSS, Positive and Negative Syndrome Scale; SD, standard deviation.

patients that required a dose adjustment, majority (71 [78.9\%] patients) of adjustments were due to insufficient efficacy. Concomitant psychotropic medications were used by 182 $(85.8 \%)$ patients. Overall, $59(27.8 \%)$ patients took oral atypical antipsychotics while $13(16.1 \%)$ patients were on oral typical antipsychotics. The most commonly used atypical antipsychotics were risperidone (34 [16.0\%] patients) and paliperidone (14 [6.6\%] patients), whereas the most frequently used typical antipsychotics were chlorpromazine and haloperidol (4 [1.9\%] patients each). Lorazepam (112 [52.8\%] patients) was the most commonly used benzodiazepine. The most commonly used class of concomitant psychotropic medication started after day 1 was benzodiazepines (107 [50.0\%] patients) and anti-EPS agents (51 [24.1\%] patients).

\section{Efficacy outcomes}

\section{Primary efficacy endpoint}

In the ITT analysis set, the mean (SD) change in the PANSS total score from baseline (90.0 [17.41]) was significant at day 4
(-6.1 [9.27]; 95\% confidence interval [CI]: $-7.38,-4.85$; $P<0.001)$, and continued to improve until the week 13 endpoint (-23.9 [23.24]); 95\% CI: $-27.10,-20.78 ; P<0.001)$ (Figure 2). The lower 95\% CI at week 13 did not include the change of 6 units in the PANSS total score. The mean (SD) change from baseline in the PANSS total score for the perprotocol set was $-6.1(9.53)(95 \% \mathrm{CI}:-7.51,-4.67 ; P<0.001)$ at day 4, and -22.4 (23.28) (95\% CI: $-25.89,-18.99$; $P<0.001)$ at the week 13 endpoint, which was similar to that of the ITT population.

For the post hoc confirmatory analysis of the efficacy population ( $\mathrm{N}=148)$, excluding patients who received concomitant antipsychotic medication after day $1(n=64)$, the mean (SD) change in the PANSS total score from baseline at day 4 was -5.6(9.18) (95\% CI: -7.09, -4.07; $P<0.001)$, and -21.9 (23.51) (95\% CI: $-25.74,-18.05 ; P<0.001)$ at the week 13 endpoint.

\section{Secondary efficacy endpoints}

The $40 \%$ PANSS response rate was achieved in $53.3 \%$ (112/210) patients at week 13 (Figure 3). Other secondary endpoints including CGI-S, PSP, each PANSS subscale and Marder Factor scores improved significantly from baseline to the endpoint $(P<0.001$ for all; Table 2$)$. At week 13, $58.0 \%$ of patients were categorized as mild/very mild/not ill, showing improvement in the CGI-S over time. The mean (SD) change in the CGI-S score from baseline to week 13 was -1.3 (1.33) (95\% CI: $-1.55,-1.11 ; P<0.001)$ for the post hoc confirmatory analysis of the efficacy population, which was similar to that of the overall ITT analysis. Based on the PSP scores, the percentage of patients categorized as poor functioning decreased from $23.6 \%$ to $6.0 \%$, varying functioning decreased from $75.5 \%$ to $64.1 \%$, and mild functioning

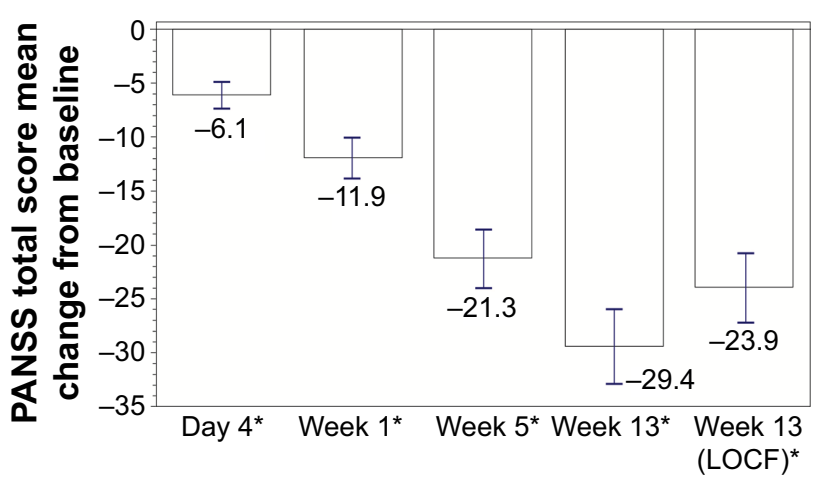

Figure 2 Mean $( \pm 2$ SE) change in PANSS total score from baseline at each timepoint, and at week 13 (ITT analysis set).

Note: $* P<0.001$ for change from baseline using paired $t$-test.

Abbreviations: ITT, intent-to-treat; LOCF, last observation carried forward; PANSS, Positive and Negative Syndrome Scale; SE, standard error. 


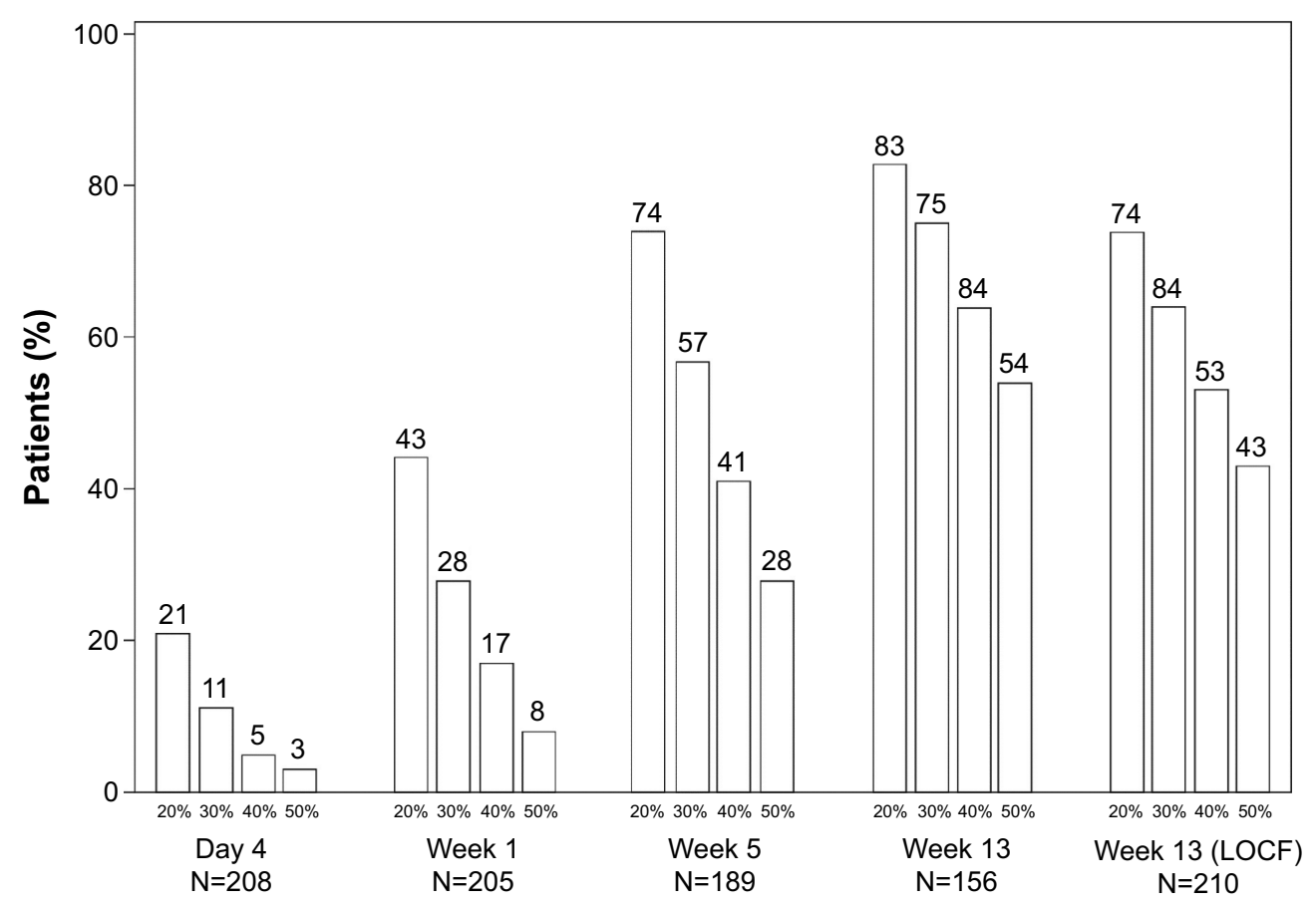

Figure 3 PANSS responder rates at each timepoint and at week 13 (ITT analysis set).

Abbreviations: ITT, intent-to-treat; LOCF, last observation carried forward; PANSS, Positive and Negative Syndrome Scale.

increased from $0.9 \%$ to $29.9 \%$, at week 13 . For the post hoc confirmatory analysis of efficacy, the PSP score significantly $(P<0.001)$ improved from baseline to week 13 (18.0 [17.56]), which was similar to that of the overall ITT analysis.

The rate of discontinuation, measured as the duration (days) of patients' hospitalization at the time of enrollment into the study, was a mean (SD) of 38.0 (27.47) days. The majority of patients (n [\%]) were hospitalized for a duration in the range of 29-56 days (55 [25.9\%] patients), and
57-91 days (54 [25.5\%] patients), followed by $8-14$ days (40 [18.9\%] patients) and 15-28 days (38 [17.9\%] patients).

Median time-to-ready-for-hospital-discharge (days since admission date) was 40 days. At week 13, 133/212 (62.7\%) patients were ready for hospital discharge (including RDQ assessment and actual hospital discharge). Overall, 102 (48.1\%) patients were discharged after completing the RDQ, whereas 42 (19.8\%) patients were discharged before completing the RDQ.

Table 2 Change from baseline to each timepoint, and at week 13 in the secondary endpoints (ITT analysis set; LOCF)

\begin{tabular}{|c|c|c|c|c|c|c|}
\hline \multirow[t]{2}{*}{ Parameter } & \multirow{2}{*}{$\begin{array}{l}\text { Baseline } \\
\text { mean (SD) }\end{array}$} & \multicolumn{5}{|c|}{ Mean (SD) change from baseline to } \\
\hline & & Day 4* & Week I* & Week $5^{*}$ & Week 9* & Week I3* \\
\hline CGI-S score & $4.9(0.79)$ & ND & $-0.6(0.73)$ & $-1.1(1.06)$ & $-1.3(1.28)$ & $-1.4(1.33)$ \\
\hline PSP score & $42.8(13.14)$ & ND & ND & ND & ND & $18.8(17.56)$ \\
\hline \multicolumn{7}{|l|}{ PANSS subscales score } \\
\hline Positive subscale score & $22.5(6.53)$ & $-2.1(3.66)$ & $-4.1(5.21)$ & $-6.4(7.08)$ & ND & $-7.7(7.91)$ \\
\hline Negative subscale score & $23.1(7.19)$ & $-1.3(2.46)$ & $-2.3(3.78)$ & $-4.1(5.74)$ & ND & $-5.2(6.82)$ \\
\hline General psychopathology score & $44.4(9.87)$ & $-2.8(5.29)$ & $-5.6(7.67)$ & $-8.9(10.46)$ & ND & $-11.0(12.20)$ \\
\hline \multicolumn{7}{|l|}{ PANSS Marder factor scores } \\
\hline Positive symptoms & $26.7(6.27)$ & $-1.7(3.37)$ & $-3.6(4.63)$ & $-6.2(6.66)$ & ND & $-7.7(7.87)$ \\
\hline Negative symptoms & $22.7(7.38)$ & $-1.3(2.56)$ & $-2.3(3.99)$ & $-4.0(5.95)$ & ND & $-5.1(7.08)$ \\
\hline Disorganized thoughts & $19.9(5.44)$ & $-1.2(2.39)$ & $-2.3(3.46)$ & $-3.9(4.74)$ & ND & $-5.0(5.8 I)$ \\
\hline $\begin{array}{l}\text { Uncontrolled hostility and } \\
\text { excitement }\end{array}$ & II.2 (4.II) & $-1.2(2.78)$ & $-2.4(3.53)$ & $-3.5(5.01)$ & ND & $-3.9(5.08)$ \\
\hline Anxiety/depression & $9.4(4.07)$ & $-0.7(\mathrm{I} .8 \mathrm{I})$ & $-1.4(2.8 \mathrm{I})$ & $-1.9(3.19)$ & ND & $-2.3(3.47)$ \\
\hline
\end{tabular}

Note: $* P<0.001$ for change from baseline using paired $t$-test for all parameters.

Abbreviations: CGI-S, Clinical Global Impression-Severity; ITT, intent-to-treat; LOCF, last observation carried forward; ND, not determined; PANSS, Positive and Negative Syndrome Scale; PSP, personal and social performance; SD, standard deviation. 


\section{Safety outcomes}

Overall, 139 (65.6\%) patients experienced at least one TEAE (Table 3) with 94 (44.3\%) patients experiencing a TEAE possibly-related to the study treatment. No deaths occurred in the study. Fourteen (6.6\%) patients experienced a serious TEAE. Four patients had treatment-related serious TEAEs, which were sinus bradycardia $(1.4 \%)$, and worsening of schizophrenia $(0.5 \%)$. Overall, $10(4.7 \%)$ patients discontinued the study due to a TEAE. The EPS-related TEAEs were reported in $37(17.5 \%)$ patients, and the most common ( $\geq 2 \%$ ) TEAEs were tremor (11 [5.2\%] patients), akathisia (10 [4.7\%] patients), extrapyramidal disorder (7 [3.3\%] patients), and dystonia (5 [2.4\%] patients). Prolactin-related TEAEs (29 [13.7\%] patients) were reported in 16 women (15.4\%), and 13 men (12.0\%). Hyperprolactinemia was the most common prolactin-related TEAE reported in $25(11.8 \%)$ patients. Amenorrhea, galactorrhea, delayed menstruation, and erectile dysfunction were each reported in one $(0.5 \%)$ patient. Diabetes mellitus was reported in $1(0.5 \%)$ patient, was mild and not related to the treatment, and did not require a dose adjustment. Overall, mean (SD) increase in the weight was $1.1(3.73) \mathrm{kg}$, with $\geq 7 \%$ increase in weight observed in $26(14.6 \%)$ patients, and $\geq 7 \%$ decrease in weight in $5(2.8 \%)$ patients.

Table 3 Most common ( $\geq 2 \%$ patients) TEAEs (ITT analysis set)

\begin{tabular}{ll}
\hline Parameter & Overall (N=2 I 2) \\
& $\mathbf{n}(\%)$ \\
\hline Patients with at least one TEAE $^{\text {Hyperprolactinemia }}{ }^{\text {a }}$ & $139(65.6)$ \\
Constipation & $25(1 \mathrm{I} .8)$ \\
Nasopharyngitis & $19(9.0)$ \\
Insomnia & $18(8.5)$ \\
Weight increased & $17(8.0)$ \\
Tremor & $16(7.5)$ \\
Akathisia & $11(5.2)$ \\
Upper respiratory tract infection & $10(4.7)$ \\
Injection site pain & $10(4.7)$ \\
Sinus bradycardia & $9(4.2)$ \\
Anxiety & $9(4.2)$ \\
Schizophrenia & $9(4.2)$ \\
Extrapyramidal disorder & $8(3.8)$ \\
Agitation & $7(3.3)$ \\
Diarrhea & $6(2.8)$ \\
Hypokalemia & $6(2.8)$ \\
Hepatic function abnormal & $6(2.8)$ \\
Dystonia & $5(2.4)$ \\
\hline Notes & $5(2.4)$ \\
\hline
\end{tabular}

Notes: aHyperprolactinemia TEAEs also include the preferred terms "blood prolactin increased" and "blood prolactin abnormal". The percentages are based on the number of patients in the ITT population. Each patient is counted only once within each system organ class and within each preferred term.

Abbreviations: ITT, intent-to-treat; TEAE, treatment-emergent adverse event.

\section{Discussion}

This open-label, Phase IV study (PREVAIL) demonstrated the efficacy of PP once-monthly injection as determined by the primary efficacy measure, ie, change in the PANSS total score from baseline to week 13, in Asian patients with an acute exacerbation of schizophrenia, within 4 weeks of hospitalization. The significant improvement in overall symptoms (improvement of -23.9 from the baseline in the PANSS total score, at week 13 LOCF) confirms the results of the earlier studies conducted in Asian hospitalized, ${ }^{24}$ and Asian or non-Asian ambulatory patients with acute exacerbated schizophrenia. ${ }^{7,33-36}$ Though data for an active comparator or placebo group were not assessed, the significant reduction in the PANSS total score is noteworthy with the 13-week PP treatment.

Patients were hospitalized for a mean duration of 38 days, with the majority being treated with oral antipsychotics before enrollment. They were switched to PP on day 1, and majority $(75.9 \%)$ of the patients received all four injections. A rapid onset of action of $\mathrm{PP}$ was demonstrated as treatment effectiveness was observed by day 4 , with improvements continuing at day 8 . These findings support the rapid symptom improvement after the day 1 deltoid injection of PP $234 \mathrm{mg}$ (150 mg eq) in these patients, which is consistent with earlier studies. ${ }^{12,34,37-39}$ The fast onset of action of PP is unique among the LAIs, and thus provides an optimal treatment option for acute exacerbation; hence, the hospitalized patients can be switched from their oral antipsychotic regimen to monthly PP injections, even during acute illness.

In this study, the major protocol deviations included "received excluded concomitant treatment" in $12.7 \%$ of patients; however, improvement in the PANSS total score was similar for both the ITT and per-protocol populations, which demonstrate a minimal effect of excluded concomitant medications on the conclusions. Similarly, the findings of the post hoc confirmatory analysis of efficacy were consistent with the overall ITT analysis, which shows no or minimal effect of concomitant antipsychotic medication.

A significant reduction in the severity of illness was seen consistently over time as measured by the CGI-S. The $40 \%$ PANSS responder rate $(53 \%)$ was considered to be clinically meaningful based on a similar improvement observed in the CGI-S scores, and this interpretation is supported by the published literature. ${ }^{40-42}$

Patients' daily function, measured by the PSP scale, showed a statistically significant and clinically meaningful improvement, which was consistent over the course of the treatment. These improvements can be translated into a 
potential reduction in the burden of patients on caregivers, society, and health care systems, and therefore, can be considered as meaningful and important in the context of benefits of treatment; a prior mediation analysis done with paliperidone ER showed that the improvement in social functioning can be independent, and above and beyond that of the symptom improvement. ${ }^{43}$

The robustness of this result is supported by the findings of consistent symptom improvement across all the efficacy measures used (PANSS and CGI-S), and also by the improved functioning outcome measured by the PSP scale.

The findings of the current study are further supported by the results from the similar PALMFlexS C study, in which patients with acute symptoms of schizophrenia who were switched to PP from oral antipsychotics showed similar efficacy results for the mean change of CGI-S ( -1.4 vs -1.5 , respectively), and PSP (18.8 vs 19.0, respectively) scores, though the efficacy differed for the mean change in the PANSS total score ( -23.9 vs -31.0 , respectively). Overall, a greater improvement in the PANSS subscales, and Marder factor scores, except hostility/excitement factor score, was observed in the PALMFlexS C study. The longer duration of drug administration is likely to have contributed to the greater improvements in the primary and secondary endpoints, as noted in the PALMFlexS C study (6 months vs 13 weeks). ${ }^{44}$

In this study, median time to ready for hospital discharge was 40 days since the admission date, and 35 days since the first injection date. Over time, a trend toward an increase in the number of patients ready for discharge was observed. At week 13, 62.7\% (LOCF) patients were ready for hospital discharge. Very few patients $(3.7 \%)$ had a subsequent relapse requiring rehospitalization. Another study demonstrated that PP significantly reduced the hospitalization duration in patients with recent-onset schizophrenia, in a 12-month mirror analysis (mean change from 74.3 to 18.9 days, $P<0.0001){ }^{24}$

Overall, treatment with PP was generally tolerable in this patient population, which is consistent with the findings from earlier studies carried out in acutely exacerbated patients. ${ }^{7,33,35}$ Incidence of adverse events appeared similar between this study and the PALMFlexS C study for $\geq 1$ TEAEs $(65.6 \%$ vs $63.7 \%)$; discontinuations $(6.1 \%$ vs $9.0 \%)$ due to TEAEs in the PALMFlexS C study may be due to the longer study duration (6 months vs 13 weeks). The frequency of prolactinrelated TEAEs was higher in the present study $(13.7 \%)$ vs PALMFlexS C (5.7\%). The higher mean (SD) body weight (78.9 kg [18.7] vs $63.6 \mathrm{~kg}$ [13.15]), BMI (36.4 kg/m² [12.1] vs $23.6 \mathrm{~kg} / \mathrm{m}^{2}$ [4.24]), and number (\%) of men (125 [59\%] vs 108 [51\%]) in the PALMFlexS study, with a related decrease in the drug exposure, may have resulted in the lower incidence of the prolactin-related TEAEs. In addition, the racial difference $(100 \%$ Asian in the current study and mostly European in the PALMFlexS) could have also attributed to these results. However, the frequency of prolactin-related TEAEs is consistent with another study conducted in the Asia-Pacific population $(11.9 \%) .{ }^{24}$ The most common TEAEs in this current study were constipation, nasopharyngitis, blood prolactin increase, and insomnia; whereas, injection-site pain, insomnia, psychotic disorder, headache, and anxiety were the most common TEAEs observed in the PALMFlexS C study. Overall, the safety profile of PP observed in this study is consistent with those previously documented and are consistent with the class effects (dopamine D2 receptors antagonists) that are well known. ${ }^{45}$

The study limitations included an open-label design, short duration of the study, a lack of comparator group to better assess the efficacy, and minimize the possible confounding variables. Additional long-term, comparative studies are required to validate these results in order to fully understand the value of PP therapy relative to other treatment options.

\section{Conclusion}

The PREVAIL study showed that PP is beneficial for acutely ill, in-hospital patients with recent exacerbation of schizophrenia, and the patients can be switched from oral antipsychotics to monthly PP injection, even during acute illness. Overall, PP was generally tolerable without new safety signals and showed significant improvements in psychotic symptoms, social functioning, and severity of illness.

\section{Acknowledgments}

The authors thank Mr Shreekant Sharma (SIRO Clinpharm Pvt. Ltd.) for writing assistance and Dr Wendy Battisti (Janssen Research \& Development, LLC) for additional editorial assistance for the development of this manuscript. The authors also thank the participants of the study, without whom the study would never have been accomplished. The study was supported by Janssen Research \& Development, LLC. The sponsor provided a formal review of the manuscript.

\section{Author contributions}

Dr Li was the principal investigator for the study, and was also involved in the design of the study, and data interpretation. Dr Turkoz was the statistical lead and had a primary role 
in the study design, statistical data analyses, and data interpretation. Dr Zhang contributed to the conception, design, data analysis, and interpretation. All authors contributed to the data interpretation, development, and revision of this manuscript, and confirm that they have read the journal's position on issues involved in ethical publication and this report is consistent with those guidelines. All authors meet ICMJE criteria, and all those who fulfilled those criteria are listed as authors. All authors had access to the study data, provided direction and comments on the manuscript, made the final decision about where to publish these data, and approved submission to this journal.

\section{Disclosure}

$\mathrm{Dr}$ Li is the chief psychiatrist at Shanghai Mental Health Center, and consultant for Boehringer Ingelheim, Roche, and AstraZeneca. Dr Turkoz is an employee of Janssen Research \& Development, Titusville, NJ, USA. Dr Zhang is an employee of Xi' an Janssen Pharmaceutical Ltd., Beijing, People's Republic of China.

\section{References}

1. Montgomery W, Liu L, Stensland MD, Xue HB, Treuer T, AscherSvanum H. The personal, societal, and economic burden of schizophrenia in the People's Republic of China: implications for antipsychotic therapy. Clinicoecon Outcomes Res. 2013;5:407-418.

2. Cahoon EK, McGinty EE, Ford DE, Daumit GL. Schizophrenia and potentially preventable hospitalizations in the United States: a retrospective cross-sectional study. BMC Psychiatry. 2013;25:13-37.

3. Lin HC, Huang CC, Chen SF, Chen YH. Increased risk of avoidable hospitalization among patients with schizophrenia. Can J Psychiatry. 2011;56(3):171-178.

4. Lehman AF, Lieberman JA, Dixon LB, et al. Practice guideline for the treatment of patients with schizophrenia, second edition. Am J Psychiatry. 2004;161(2 Suppl):1-56.

5. Casey DE. Long-term treatment goals: enhancing healthy outcomes. CNS Spectr. 2003;8(11 Suppl 2):26-28.

6. Awad AG, Voruganti LN. The burden of schizophrenia on caregivers: a review. Pharmacoeconomics. 2008;26(2):149-162.

7. Pandina GJ, Lindenmayer JP, Lull J, et al. A randomized, placebocontrolled study to assess the efficacy and safety of 3 doses of paliperidone palmitate in adults with acutely exacerbated schizophrenia. J Clin Psychopharmacol. 2010;30(3):235-244.

8. Brown MT, Bussell JK. Medication adherence: WHO cares? Mayo Clin Proc. 2011;86(4):304-314.

9. Ho PM, Bryson CL, Rumsfeld JS. Medication adherence: its importance in cardiovascular outcomes. Circulation. 2009;119(23): 3028-3035.

10. Law MR, Soumerai SB, Ross-Degnan D, Adams AS. A longitudinal study of medication nonadherence and hospitalization risk in schizophrenia. J Clin Psychiatry. 2008;69(1):47-53.

11. Weiden PJ, Kozma C, Grogg A, Locklear J. Partial compliance and risk of rehospitalization among California Medicaid patients with schizophrenia. Psychiatr Serv. 2004;55(8):886-891.

12. Alphs L, Bossie CA, Sliwa JK, Ma YW, Turner N. Onset of efficacy with acute long-acting injectable paliperidone palmitate treatment in markedly to severely ill patients with schizophrenia: post hoc analysis of a randomized, double-blind clinical trial. Ann Gen Psychiatry. 2011;10(1):12.
13. Lafeuille MH, Laliberte-Auger F, Lefebvre P, Frois C, Fastenau J, Duh MS. Impact of atypical long-acting injectable versus oral antipsychotics on rehospitalization rates and emergency room visits among relapsed schizophrenia patients: a retrospective database analysis. BMC Psychiatry. 2013;13:221.

14. Lambert T, Olivares JM, Peuskens J, et al. Effectiveness of injectable risperidone long-acting therapy for schizophrenia: data from the US, Spain, Australia, and Belgium. Ann Gen Psychiatry. 2011;10:10.

15. Velligan DI, Weiden PJ, Sajatovic M, et al. The expert consensus guideline series: adherence problems in patients with serious and persistent mental illness. J Clin Psychiatry. 2009;70(Suppl 4):1-46; quiz $47-48$.

16. Invega ${ }^{\circledR}$ Sustenna ${ }^{\circledR}$ (paliperidone palmitate extended-release injectable suspension) [package insert]. Titusville, NJ: Janssen Pharmaceuticals, Inc.; 2014.

17. Citrome L. Paliperidone palmitate - review of the efficacy, safety and cost of a new second-generation depot antipsychotic medication. Int $J$ Clin Pract. 2010;64(2):216-239.

18. Hoy SM, Scott LJ, Keating GM. Intramuscular paliperidone palmitate. CNS Drugs. 2010;24(3):227-244.

19. Kramer M, Simpson G, Maciulis V, et al. Paliperidone extended-release tablets for prevention of symptom recurrence in patients with schizophrenia: a randomized, double-blind, placebo-controlled study. J Clin Psychopharmacol. 2007;27(1):6-14.

20. Potkin SG, Gharabawi GM, Greenspan AJ, et al. A double-blind comparison of risperidone, quetiapine and placebo in patients with schizophrenia experiencing an acute exacerbation requiring hospitalization. Schizophrenia Res. 2006;85(1):254-265.

21. Samtani MN, Gopal S, Gassmann-Mayer C, Alphs L, Palumbo JM. Dosing and switching strategies for paliperidone palmitate. CNS Drugs. 2011;25(10):829-845.

22. Owen R. Paliperidone palmitate injection: its efficacy, safety and tolerability in schizophrenia. Drugs Today (Barc). 2010;46(7):463-471.

23. Pandina G, Lane R, Gopal S, et al. A double-blind study of paliperidone palmitate and risperidone long-acting injectable in adults with schizophrenia. Prog Neuropsychopharmacol Biol Psychiatry. 2011;35(1):218-226.

24. Zhang F, Si T, Chiou CF, et al. Efficacy, safety, and impact on hospitalizations of paliperidone palmitate in recent-onset schizophrenia. Neuropsychiatr Dis Treat. 2015;11:1-12.

25. Bugarski-Kirola D, Wang A, Abi-Saab D, Blattler T. A phase II/III trial of bitopertin monotherapy compared with placebo in patients with an acute exacerbation of schizophrenia - results from the CandleLyte study. Eur Neuropsychopharmacol. 2014;24(7):1024-1036.

26. De la Gandara J, San Molina L, Rubio G, Rodriguez-Morales A, Hidalgo Borrajo R, Buron JA. Experience with injectable long-acting risperidone in long-term therapy after an acute episode of schizophrenia: the SPHERE Study. Expert Rev Neurother. 2009;9(10): 1463-1474.

27. Meltzer HY, Cucchiaro J, Silva R, et al. Lurasidone in the treatment of schizophrenia: a randomized, double-blind, placebo- and olanzapinecontrolled study. Am J Psychiatry. 2011;168(9):957-967.

28. Marder SR, Davis JM, Chouinard G. The effects of risperidone on the five dimensions of schizophrenia derived by factor analysis: combined results of the North American trials. J Clin Psychiatry. 1997;58(12): 538-546.

29. National Institute of Mental Health. Clinical Global Impressions (CGI). In: Guy W, editor. ECDEU Assessment Manual for Psychopharmacology. Bethesda: US Department of Health, Education and Welfare; 1976:218-222.

30. Morosini PL, Magliano L, Brambilla L, Ugolini S, Pioli R. Development, reliability and acceptability of a new version of the DSM-IV Social and Occupational Functioning Assessment Scale (SOFAS) to assess routine social functioning. Acta Psychiatr Scand. 2000;101(4): 323-329.

31. Hogarty GE, Ulrich R. The discharge readiness inventory. Arch Gen Psychiatry. 1972;26(5):419-426. 
32. Potkin SG, Gharabawi GM, Greenspan AJ, et al. Psychometric evaluation of the Readiness for Discharge Questionnaire. Schizophr Res. 2005;80(2):203-212.

33. Gopal S, Hough DW, Xu H, et al. Efficacy and safety of paliperidone palmitate in adult patients with acutely symptomatic schizophrenia: a randomized, double-blind, placebo-controlled, dose-response study. Int Clin Psychopharmacol. 2010;25(5):247-256.

34. Kramer M, Litman R, Hough D, et al. Paliperidone palmitate, a potential long-acting treatment for patients with schizophrenia. Results of a randomized, double-blind, placebo-controlled efficacy and safety study. Int J Neuropsychopharmacol. 2010;13(5):635-647.

35. Nasrallah HA, Gopal S, Gassmann-Mayer C, et al. A controlled, evidence-based trial of paliperidone palmitate, a long-acting injectable antipsychotic, in schizophrenia. Neuropsychopharmacology. 2010;35(10):2072-2082.

36. Li H, Rui Q, Ning X, Xu H, Gu N. A comparative study of paliperidone palmitate and risperidone long-acting injectable therapy in schizophrenia. Prog Neuropsychopharmacol Biol Psychiatry. 2011;35(4):1002-1008.

37. Fu DJ, Bossie CA, Kern Sliwa J, Ma YW, Alphs L. Paliperidone palmitate versus risperidone long-acting injection in markedly-to-severely ill schizophrenia subjects: onset of efficacy with recommended initiation regimens. Clin Schizophr Relat Psychoses. 2014;8(2):101-109, 109A.

38. Bossie CA, Sliwa JK, Ma YW, Fu DJ, Alphs L. Onset of efficacy and tolerability following the initiation dosing of long-acting paliperidone palmitate: post-hoc analyses of a randomized, double-blind clinical trial. BMC Psychiatry. 2011;11:79.
39. Alphs L, Bossie CA, Fu DJ, Ma YW, Kern Sliwa J. Onset and persistence of efficacy by symptom domain with long-acting injectable paliperidone palmitate in patients with schizophrenia. Expert Opin Pharmacother. 2014;15(7):1029-1042.

40. Leucht S, Kane JM, Etschel E, Kissling W, Hamann J, Engel RR. Linking the PANSS, BPRS, and CGI: clinical implications. Neuropsychopharmacology. 2006;31(10):2318-2325.

41. Leucht S, Kane JM, Kissling W, Hamann J, Etschel E, Engel R. Clinical implications of Brief Psychiatric Rating Scale scores. Br J Psychiatry. 2005; 187:366-371.

42. Leucht S, Kane JM, Kissling W, Hamann J, Etschel E, Engel RR. What does the PANSS mean? Schizophr Res. 2005;79(2-3):231-238.

43. Hough D, Nuamah IF, Lim P, Sampson A, Gagnon DD, Rothman M. Independent effect of paliperidone extended release on social functioning beyond its effect on positive and negative symptoms of schizophrenia: a mediation analysis. J Clin Psychopharmacol. 2009;29(5): 496-497.

44. Hargarter L, Cherubin P, Bergmans P, et al. Intramuscular long-acting paliperidone palmitate in acute patients with schizophrenia unsuccessfully treated with oral antipsychotics. Prog Neuropsychopharmacol Biol Psychiatry. 2014;58c:1-7.

45. Committee for Medicinal Products for Human Use, European Medicines Agency. Guideline on Clinical Investigation of Medicinal Products, Including Depot Preparations in the Treatment of Schizophrenia. London: European Medicines Agency; 2012. Available from: http://www.ema.europa.eu/docs/en_GB/document_library/Scientific_guideline/2012/10/WC500133437.pdf. Accessed November 24, 2014.
Neuropsychiatric Disease and Treatment

\section{Publish your work in this journal}

Neuropsychiatric Disease and Treatment is an international, peerreviewed journal of clinical therapeutics and pharmacology focusing on concise rapid reporting of clinical or pre-clinical studies on a range of neuropsychiatric and neurological disorders. This journal is indexed on PubMed Central, the 'PsycINFO' database and CAS,

\section{Dovepress}

and is the official journal of The International Neuropsychiatric Association (INA). The manuscript management system is completely online and includes a very quick and fair peer-review system, which is all easy to use. Visit http://www.dovepress.com/testimonials.php to read real quotes from published authors. 